\title{
Towards a full model for ocular biomechanics, fluid dynamics, and hemodynamics
}

Lorenzo Sala ${ }^{1}$, Christophe Prud'Homme ${ }^{1}$, Marcela Szopos ${ }^{1}$, Giovanna Guidoboni ${ }^{2}$

IInstitute for Advanced Mathematical Research, Unité Mixte de Recherche 7501, National Center for Scientific Research, University of Strasbourg, Strasbourg, France; ${ }^{2}$ Department of Electrical Engineering and Computer Science, University of Missouri, Columbia, MO, USA

\section{Abstract}

This contribution presents an ongoing work to implement a patient-specific mathematical virtual simulator for the eye. The aim is to create a multiscale and multiphysics model for the description of ocular biomechanics, fluid dynamics, and hemodynamics. This instrument may serve to illustrate and estimate some clinically relevant parameters, as well as predict their spatial and temporal evolution adopting forward-looking numerical techniques.

Keywords: hybridizable discontinuous Galerkin method, multiphysics, multiscale, ocular virtual simulator

\section{Introduction}

Our purpose is to develop a patient-specific Mathematical Virtual Simulator (MVS) that can describe and quantify the interaction between biomechanics, fluid dynamics, and hemodynamics in the eye. The aim is to couple all the various physical aspects in the same model in order to have a full overview of the ocular system. To reach this ambitious goal, we have identified four main modeling steps:

1. blood circulation in the eye from a systemic viewpoint;

Correspondence: Lorenzo Sala, Université de Strasbourg, CNRS, IRMA UMR 7501, Strasbourg, France.

E-mail: sala@unistra.fr 
2. biomechanics and tissue perfusion in the optic nerve head and the lamina cribrosa; ${ }^{1}$

3. biomechanics for the sclera, retina, choroid, and cornea; and

4. fluid dynamics of the vitreous and aqueous humor.

Each step is naturally coupled with the others, giving rise to challenging problems from both the modeling and computational perspectives. In this abstract, we focus our attention on the second and third steps.

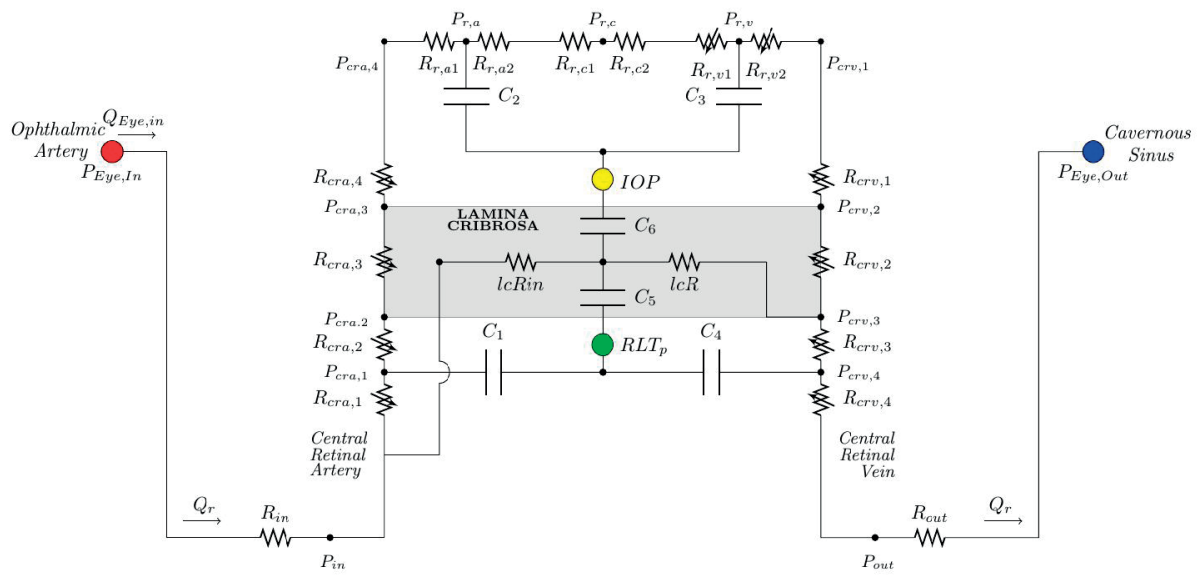

Fig. 1. Circuit-based model retinal and retrobulbar flow.

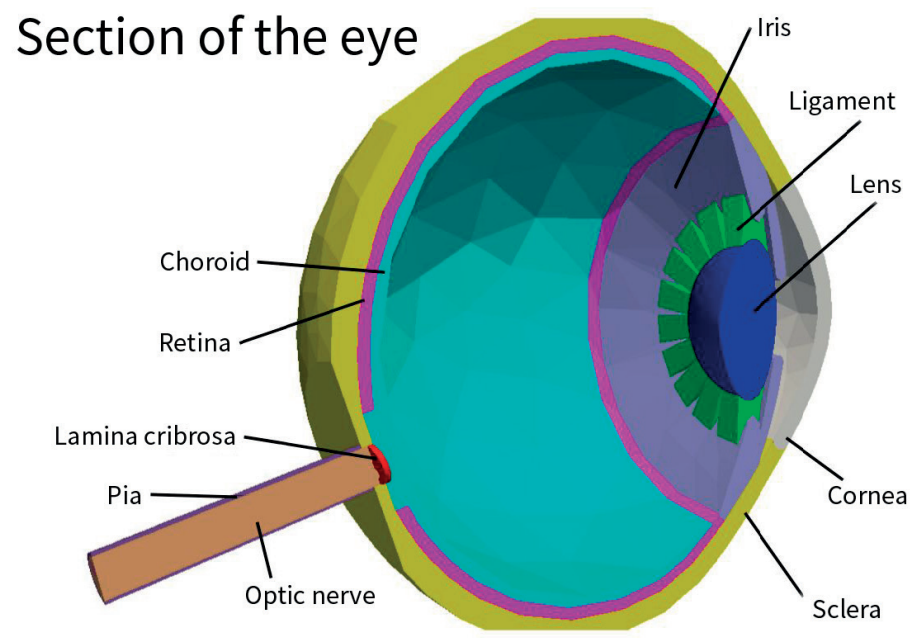

Fig. 2. The 3-D geometry generated with Salome. 


\section{Model and methods}

The MVS has a multiscale architecture that aims at preserving the natural systemic features of blood circulation, while providing detailed views on sites of particular interest from the clinical viewpoint, such as the lamina cribrosa. Thus, MVS combines:

1. a circuit-based model for blood flow in the retinal vasculature, central retinal artery (CRA), and central retinal vein (CRV);

2. a 3-D porous media model for the perfusion of the lamina cribrosa; and

3. a 3-D isotropic elastic model for the biomechanics in the lamina cribrosa, retina, choroid, sclera, and cornea.

Figure 1 illustrates the circuit-based model in which we are substituting the lumped-parameters description of the lamina with a poroelastic spatial distribution model of the lamina. The elastic system of the lamina is also influenced by the biomechanics of the sclera, retina, choroid, and cornea. The novelty of the methods in the current work comes from two complementary perspectives. On the one hand, from a clinical perspective, the model inputs (e.g., blood pressure, intraocular pressure, and ocular geometry) are easily accessible with standard instruments and can therefore be tailored to patient-specific conditions. In particular, starting from an initial computer-aided design (CAD) geometrical model, the structure of the geometry is further elaborated using Salome (OPEN CASCADE SAS, Guyancourt, France) ${ }^{1}$ with the help of a Python (Python Software Foundation, The Netherlands) script that incorporates some parameterized values (e.g., thickness of the lamina cribrosa) into the final meshed geometry (Fig. 2). On the other hand, from a numerical viewpoint, the problem involves a non-trivial coupling between biomechanics and hemodynamics in the tissue of the lamina cribrosa, which calls for:

1. high accuracy in the approximation for both primal variables (i.e., displacement and pressure) and dual variables (i.e., stress and perfusion velocity); and

2. integral boundary conditions to account for the coupling between 0-D and 3-D model components.

\subsection{Coupling between biomechanics and hemodynamics}

The two-way coupling between biomechanics and hemodynamics contributing to tissue perfusion in the lamina cribrosa is taken into account by means of the following poroelastic model:

$$
\begin{aligned}
& \nabla \cdot \underline{\underline{\sigma}}=f_{e l} \\
& \underline{\underline{\sigma}}=\underline{\underline{\sigma}}_{e l}-p \underline{\underline{I}}
\end{aligned}
$$




$$
\begin{aligned}
& \underline{\underline{\sigma}}_{e l}=\mu\left(\nabla \underline{u}+\nabla^{\top} \underline{u}\right)+\lambda(\nabla \cdot \underline{u}) \underline{\underline{I}} \\
& \nabla \cdot u t+\nabla \cdot \underline{v}=f_{f l} \\
& \underline{v}+\mathrm{k} \nabla p=0
\end{aligned}
$$

where $\underline{\sigma}$ is the total stress tensor, $\mu$ is the solid displacement, $\rho$ is the fluid pressure, $v$ is the discharge velocity, $\lambda$ and $\mu$ are the elastic parameters, $k$ is the permeability, and $\boldsymbol{f}_{e l}$ and $f_{f l}$ are volumetric sources of linear momentum and fluid mass, respectively. The poroelastic system, illustrated in Equations (1-5), has been already studied and presented in Causin et al. ${ }^{2}$ and Prada. ${ }^{3}$ Here, we highlight the importance of the two terms in red, since they are responsible for the biomechanical/hemodynamical coupling. Indeed, the presence of capillaries within the lamina collagen beams may influence the biomechanical behavior of the tissue, as expressed in Equation (2). In turn, structural deformations may induce local changes in blood flow, as expressed in Equation (4).

\subsection{Hybridizable discontinuous Galerkin method and integral boundary con- dition}

To numerically solve the coupled system of Equations (1-5), we implemented a hybridizable discontinuous Galerkin (HDG) method $^{4}$ in the multiphysics open-source platform Feel++ (Cemosis; Strasbourg, France). ${ }^{5}$ The HDG method has several attractive features:

1. it provides optimal approximation of both primal (pressure, displacement) and dual (velocity, stress) variables;

2. it requires less globally coupled degrees of freedom than DG methods of comparable accuracy using static condensation; and

3. it allows local element-by-element post-processing to obtain new approximations with enhanced accuracy and conservation properties.

Furthermore, to handle the multiscale nature of this problem, we coupled the 3-D system of partial differential Equations (1-5) with a 0-D circuit model implemented in OpenModelica (Open Source Modelica Consortium; Linköping, Sweden). The complex coupling between the HDG system and the circuit has been achieved using integral boundary condition (IBC) and a time-splitting energy-based scheme in the spirit of a recent method presented in Carichino et al. ${ }^{6}$ The combination of IBC with the high accuracy in dual variables of the HDG method revealed to be determinant for the proper resolution of the coupled multiscale problem. A detailed explanation of this procedure can be found in Sala et al. ${ }^{7}$ 

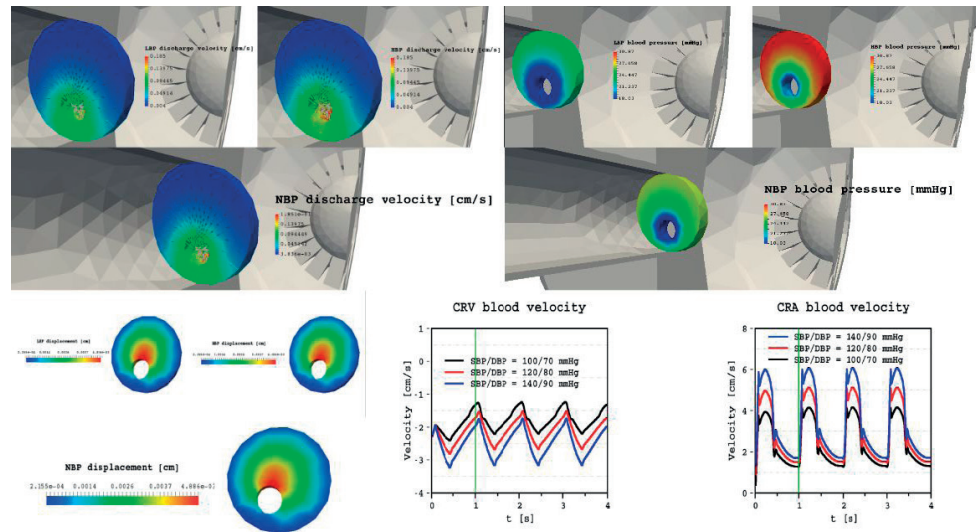

Fig. 3. Lamina cribrosa and CRA/CRV results simulated via MVS.

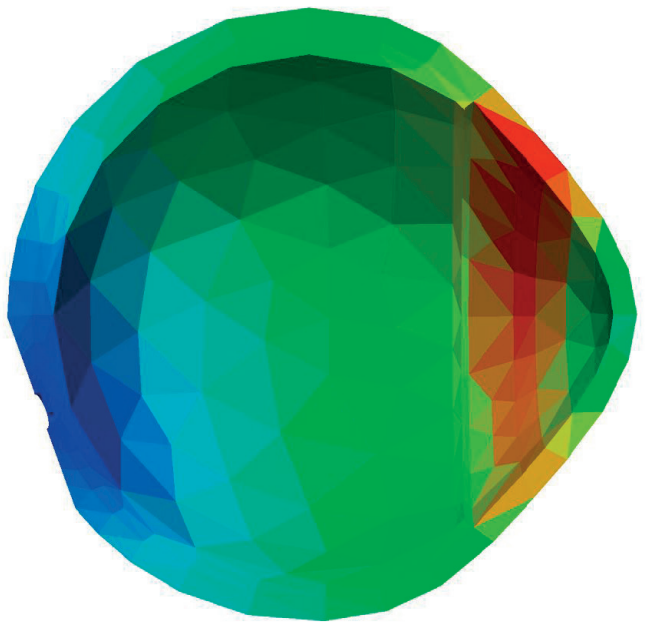

Displacement

High

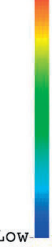

Fig. 4. Qualitative displacement on retina, choroid, sclera, and cornea simulated via MVS.

\section{Results}

Simulated distributions of velocities and pressure within the lamina, and of blood velocity within the CRA and CRV obtained via the MVS (see Fig. 3) exhibit a satisfactory agreement with reported experimental data, ${ }^{8-10}$ with a special focus on the blood pressure computed on the lateral boundary of the lamina, where we have applied the integral interface condition between the $0-D$ and the 3-D models. 
Moreover, some outcomes (Fig. 3, two bottom right figures) of MVS can be directly compared to those obtained via direct imaging modalities. ${ }^{11}$

In Figure 4, we show an overview of the qualitative behavior of the displacement for the lamina, retina, choroid, sclera, and cornea.

\section{Conclusion}

In conclusion, MVS combines innovative and complex methods such as integral boundary condition for HDG in order to be able to address the increasing demand of information on parts of the eye that are not easily accessible with standard research methods, such as those regarding the perfusion of the lamina cribrosa in the optic nerve head. Furthermore, the proposed model develops an interesting multiphysics and multiscale approach to connect different characters such as biomechanics and hemodynamics.

\section{Acknowledgements}

This work has been partially supported by the Ministry of Higher Education and Research (France), National Science Foundation (USA) DMS-1224195, a grant from Research to Prevent Blindness (RPB, NY, USA), the Chair Gutenberg funds of the Cercle Gutenberg (France), the Center for Modeling and Simulation in Strasbourg (Cemosis), and the Labex IRMIA (University of Strasbourg, France). This project has received funding from the European Union's Horizon 2020 Programme for Research and Innovation under grant agreement No. 731063.

\section{References}

1. Sala L, Prudhomme C, Prada D, et al. Patient-specific virtual simulator of tissue perfusion in the lamina cribrosa. In: Annual Meeting of the Association for Research in Vision and Ophthalmology. 7-11 May 2017. Baltimore, MD, USA.

2. Causin P, Guidoboni G, Harris A, Prada D, Sacco R, Terragni S. A poroelastic model for the perfusion of the lamina cribrosa in the optic nerve head. Math Biosci. 2015; 257:33-41.

3. Prada D. A hybridizable discontinuous Galerkin method for nonlinear porous media viscoelasticity with applications in ophthalmology. PhD thesis at IUPUI. 2016. Indianapolis (IN) USA.

4. Cockburn B, Gopalakrishnan J, Lazarov R. Unified hybridization of discontinuous Galerkin, mixed, and continuous Galerkin methods for second order elliptic problems. SIAM J Numer Anal. 2009;47(2):1319-1365.

5. Prud'Homme C, Chabannes V, Doyeux V, Ismail M, Samake A, Pena G. Feel++: A Computational Framework for Galerkin Methods and Advanced Numerical Methods ESAIM: Proceedings, EDP Sciences. 2012; 38: pp. 429-455. doi: 10.1051/proc/201238024 
6. Carichino L, Guidoboni G, Szopos M. Operator splitting approach for coupling Stokes flow and nonlinear systems of ODEs. In: 7th International Conference on Computational Methods for Coupled Problems in Science and Engineering. 2017. Rhodes, Greece.

7. Sala L, Hild R, Prud'Homme C, et al. An implementation of HDG methods with Feel++. Application to problems with integral boundary condition. In preparation. 2017.

8. Yan DB, Coloma FM, Metheetrairut A, Trope GE, Heathcote JG, Ethier CR. Deformation of the lamina cribrosa by elevated intraocular pressure. Br J Ophthalmol. 1994;78(8):643-648.

9. Sigal IA, Flanagan JG, Tertinegg I, Ethier CR. Finite element modeling of optic nerve head biomechanics. Invest Ophthalmol Vis Sci. 2004;45(12):4378-4387.

10. Guidoboni G, Harris A, Cassani S, et al. Intraocular pressure, blood pressure, and retinal blood flow autoregulation: A mathematical model to clarify their relationship and clinical relevance. Invest Ophthalmol Vis Sci. 2014;55(7):4105-4118.

11. Williamson TH, Harris A. Color Doppler ultrasound imaging of the eye and orbit. Surv Ophthalmol. 1996;40(4):255-267. 